\title{
Performance bounds on optimal fixed prices
}

\author{
Alper Şen* \\ Department of Industrial Engineering, Bilkent University, Bilkent, Ankara, 06800, Turkey
}

\section{A R T I C L E I N F O}

\section{Article history:}

Received 19 October 2012

Received in revised form

28 June 2013

Accepted 29 June 2013

Available online 17 July 2013

\section{Keywords:}

Dynamic pricing

Revenue management

Yield management

Heuristics

\begin{abstract}
A B S T R A C T
We consider the problem of selling a fixed stock of items over a finite horizon when the buyers arrive following a Poisson process. We obtain a general lower bound on the performance of using a fixed price rather than dynamically adjusting the price. The bound is $63.21 \%$ for one unit of inventory, and it improves as the inventory increases. For the one-unit case, we also obtain tight bounds: $89.85 \%$ for the constantelasticity and $96.93 \%$ for the linear price-response functions.
\end{abstract}

(c) 2013 Elsevier B.V. All rights reserved.

\section{Introduction}

We consider the problem of selling a fixed stock of items over a finite horizon. Potential customers arrive following a Poisson process and decide whether to buy the product or not based on the posted price and their maximum willingness-to-pay. The seller knows the probability distribution of the customers' maximum willingness-to-pay and adjusts the price as a function of remaining time in the horizon so as to maximize his/her expected revenue. It is assumed that each customer has a chance to buy the product only at his/her (first) arrival, i.e., the seller cannot recall a customer who already decided not to buy the product (no recalls) or a customer cannot return later to buy the product at a lower price (no strategic consumer behavior).

Kincaid and Darling [7] were the first to study this problem, which they call "inventory pricing problem", and to characterize the function that the seller should use to update the price over the horizon. Elfving [3] notices that the special case of a single unit of inventory is similar to what is known as the "secretary problem" or the "best choice problem" (see [5]), except that the arrivals (of candidates, i.e., potential buyers in our problem) follow a point process and the decision maker has distributional information about the desirability of candidates. Elfving [3] investigates the use of a "critical curve" to govern the decision maker's decision to accept or reject an arriving candidate. This curve essentially plays the role of price (as a function of time) in our problem; if an arriving

\footnotetext{
* Tel.: +90 312290 1539; fax: +90 3122664054

E-mail address: alpersen@bilkent.edu.tr.
}

candidate's desirability (or offer) is larger than or equal to the value of the function at the candidate's arrival time, the candidate is accepted and the process stops. Elfving [3] characterizes the unique curve in the presence of discounting. Stadje [10] derives conditions that are equivalent to those obtained in [7] for pricing a single or multiple units of inventory.

The generalized version of this problem for multiple units has been studied in a seminal work by Gallego and van Ryzin [6] and subsequently by Bitran and Mondschein [2]. Since then, dynamic pricing in the presence of inventory considerations has been the subject of an extensive literature in Operations Research (see [11,4] for reviews). An important question is whether dynamic pricing offers substantial revenue improvements over keeping the price constant over the horizon. The main finding in [6] is that using a constant price at a level that is determined by the solution of the deterministic version problem has a bounded worst performance and is asymptotically optimal as the expected demand and starting inventory go to infinity. Note that solving the deterministic version of the problem to obtain the fixed price is a heuristic even for static pricing; one can also optimize among all possible prices. The numerical results in [6] show that, even for smaller-size problems, optimal fixed prices lead to a very good performance. In fact, under an exponential price-response function, the worst performance reported in that article is $94.5 \%$. The authors state that they have never observed a suboptimality gap more than $7 \%$ (p. 1009). Zhao and Zheng [12] find that the constant-demand elasticity priceresponse function leads to larger gaps than the exponential priceresponse function. The worst performance that they report is $92.74 \%$ (the price is selected from a set of 11 alternatives in this case). The worst performance reported in an extensive numerical 
study in [9] that involves linear, logit, and exponential priceresponse functions is $93.43 \%$. For a discrete-time version of the problem, Bearden et al. [1] report that the worst performance in their numerical experiments is $94 \%$.

In this paper, we develop theoretical lower bounds for the worst performance of fixed pricing heuristics. For multiple units of starting inventory, we obtain a lower bound for the fixed pricing heuristic that is independent of the price-response function and the length of the selling season. For a single unit of inventory, we obtain tight bounds for constant-elasticity and linear priceresponse functions. These price-response functions are widely used in theory and practice, and they represent the two extreme forms for the potential benefits of dynamic pricing. Under the constant-elasticity price-response function, potential buyers have the same sensitivity to price changes at all price levels. This allows the seller to test higher prices in the beginning of the horizon since price reductions remain effective later in the horizon if needed. In contrast, under a linear price-response function, the elasticity decreases as the price is lowered over the course of the horizon. Confirming these intuitions, we show that dynamic pricing is most useful under a constant-elasticity priceresponse function and that the theoretical worst performance of optimal fixed pricing is $89.85 \%$. On the other hand, the worst performance of optimal fixed pricing under a linear price-response function is significantly higher, at $96.93 \%$. Our numerical results show that other parametric price-response functions have worst performances that are between these two bounds.

\section{Model and a general bound}

We consider a seller that needs to sell $n$ units of inventory of a product over a horizon of length $t$. Buyers arrive following a Poisson process whose rate depends only on the current price $p$ through a function $\lambda(p)$. Alternatively, one may assume that potential customers, whose reservation prices (maximum willingness-to-pay) are independently and identically distributed with a cumulative distribution function $F$, arrive with a constant rate $\gamma$, leading to a purchase rate $\lambda(p)=\gamma(1-F(p))$. The function $\lambda(p)$ is assumed to be continuous, differentiable, and decreasing in $p$ (we use the words increasing and decreasing in a non-strict sense throughout the paper). The inverse of $\lambda(p)$ is denoted by $p(\lambda)$. The unconstrained revenue rate is denoted by $r(\lambda)=\lambda p(\lambda)$, and is assumed to be concave in $\lambda$.

In the dynamic pricing problem, the firm controls the intensity at every instant by adjusting the price. The problem is to determine the pricing policy that maximizes the total expected revenue over the season, denoted by $J^{*}(n, t)$. Gallego and van Ryzin [6] show that the optimal expected revenue-to-go (and the corresponding optimal price/intensity at that instant) can be determined by solving the differential equation

$$
\frac{\partial J^{*}(n, t)}{\partial t}=\max _{\lambda}\left\{r(\lambda)-\lambda\left(J^{*}(n, t)-J^{*}(n-1, t)\right)\right\},
$$

with boundary conditions $J^{*}(n, 0)=0, \forall n$ and $J^{*}(0, t)=0, \forall t$. Equivalent characterizations are provided in [7,3,10]. Gallego and van Ryzin [6] also prove the existence of a unique solution to (1) along with monotonicity of the optimal expected revenue (and corresponding demand rates and prices) with respect to remaining time in the horizon and inventory.

Gallego and van Ryzin [6] also show that the optimal revenue for the deterministic problem in which the demand rate is a deterministic function of a given price constitutes an upper bound for the optimal revenue that can be obtained through dynamic pricing. Using the concavity of the revenue rate function, Gallego and van Ryzin [6] show that the optimal solution for the deterministic problem is to have a constant rate $\lambda^{D}=\min \left\{\lambda^{*}, \frac{n}{t}\right\}$, where $\lambda^{*}$ is the maximizer of the revenue rate function $r$. The optimal revenue of the deterministic problem is then given by

$J^{D}(n, t)=\operatorname{tp}\left(\min \left\{\lambda^{*}, \frac{n}{t}\right\}\right) \min \left\{\lambda^{*}, \frac{n}{t}\right\}$.

On the other hand, the expected revenue of keeping the demand rate fixed at $\lambda$ (or the price fixed at $p(\lambda)$ ) is given by

$J^{f}(n, t, \lambda)=p(\lambda) \mathbb{E}\left[\min \left\{n, N_{\lambda t}\right\}\right]$,

where $N_{\mu}$ is a Poisson random variable with mean $\mu$. Gallego and van Ryzin [6] suggest two heuristics for the dynamic pricing problem. In the fixed price (FP) heuristic, the demand rate is fixed at $\lambda^{D}=\min \left\{\lambda^{*}, \frac{n}{t}\right\}$, leading to an expected cost of $J^{\mathrm{FP}}(n, t)=$ $J^{f}\left(n, t, \lambda^{D}\right)$. In the optimal fixed price (OFP) heuristic, the optimal demand rate (or the price) is used, i.e., $J^{\mathrm{OFP}}(n, t)=\max _{\lambda} J^{f}(n, t, \lambda)$. Using the deterministic solution and a lower bound on $J^{\mathrm{FP}}(n, t)$, Gallego and van Ryzin [6] obtain the following result:

$\frac{J^{\mathrm{OFP}}(n, t)}{J^{*}(n, t)} \geq \frac{J^{\mathrm{FP}}(n, t)}{J^{*}(n, t)} \geq 1-\frac{1}{2 \sqrt{\min \left\{n, \lambda^{*} t\right\}}}$.

The bound in (3) approaches 1 as $n$ and $\lambda^{*} t$ approach infinity, showing the asymptotical optimality of the fixed price and optimal fixed price heuristics.

Using a different approach, we obtain the following bound, which is independent of the length of the selling season and the price-response function.

\section{Proposition 1.}

$\frac{J^{\mathrm{OFP}}(n, t)}{J^{*}(n, t)} \geq \frac{J^{\mathrm{FP}}(n, t)}{J^{*}(n, t)} \geq 1-\frac{n^{n}}{n !} e^{-n}$.

Proof. The expected revenue of using the optimal rate of the deterministic problem is given by

$J^{\mathrm{FP}}(n, t)=p\left(\min \left\{\lambda^{*}, \frac{n}{t}\right\}\right) \mathbb{E}\left[\min \left\{n, N_{\left.\left.\min \left\{\lambda^{*}, \frac{n}{t}\right\} t\right\}\right] .}\right.\right.$

Using (2) and (5), we get

$\frac{J^{\mathrm{FP}}(n, t)}{J^{D}(n, t)}=\frac{\mathbb{E}\left[\min \left\{n, N_{\min \left\{\lambda^{*} t, n\right\}}\right\}\right]}{\min \left\{\lambda^{*} t, n\right\}}$.

First note that $\mathbb{E}\left[\min \left\{s, N_{\mu}\right\}\right]=\sum_{i=0}^{s} i q(i, \mu)+s\left(1-\sum_{i=0}^{s}\right.$ $q(i, \mu))$, where $q(i, \mu)=\frac{\mu^{i} e^{-\mu}}{i !}$. Denoting $Q(i, \mu)=\sum_{k=0}^{i} q(k, \mu)$, one can easily show that $\mathbb{E}\left[\min \left\{s, N_{\mu}\right\}\right]=\mu Q(s-1, \mu)+s(1-$ $Q(s, \mu))$.

If $\lambda^{*} t>n$, we get

$$
\begin{aligned}
\frac{J^{\mathrm{FP}}(n, t)}{J^{D}(n, t)} & =\frac{\mathbb{E}\left[\min \left\{n, N_{n}\right\}\right]}{n} \\
& =\frac{n Q(n-1, n)+n(1-Q(n, n))}{n} \\
& =1-q(n, n)=1-\frac{n^{n}}{n !} e^{-n} .
\end{aligned}
$$

In order to analyze the case when $\lambda^{*} t \leq n$, we note that $\mathbb{E}\left[\min \left\{s, N_{\mu}\right\}\right] / \mu$ measures the expected fill-rate of a newsvendor who orders $s$ units and faces a Poisson demand with mean $\mu$. We can write the partial derivative of $\mathbb{E}\left[\min \left\{s, N_{\mu}\right\}\right] / \mu$ with respect to $\mu$ as the expression given in Box I where $Q_{\mu}(i, \mu)$ is the partial derivative of $Q(i, \mu)$ with respect to $\mu$. One can show that $Q_{\mu}(i, \mu)=-q(i, \mu)$ and $i q(i, \mu)=\mu q(i-1, \mu)$, leading to $\mu Q_{\mu}(s-1, \mu)=s Q_{\mu}(s, \mu)$. Using this and simplifying further, we obtain the partial derivative of $\mathbb{E}\left[\min \left\{s, N_{\mu}\right\}\right] / \mu$ with respect 


$$
\frac{\mu\left[Q(s-1, \mu)+\mu Q_{\mu}(s-1, \mu)-s Q_{\mu}(s, \mu)\right]-\mu Q(s-1, \mu)-s(1-Q(s, \mu))}{\mu^{2}},
$$

Box I.

to $\mu$ as $-s(1-Q(s, \mu)) / \mu^{2}$, which shows that $\mathbb{E}\left[\min \left\{s, N_{\mu}\right\}\right] / \mu$ is decreasing in $\mu$. This leads to

$\frac{J^{\mathrm{FP}}(n, t)}{J^{D}(n, t)}=\frac{\mathbb{E}\left[\min \left\{n, N_{\lambda^{*} t}\right\}\right]}{\lambda^{*} t} \geq \frac{\mathbb{E}\left[\min \left\{n, N_{n}\right\}\right]}{n}=1-\frac{n^{n}}{n !} e^{-n}$

for $\lambda^{*} t \leq n$. Using $J^{D}(n, t) \geq J^{*}(n, t)$ and $J^{\mathrm{OFP}}(n, t) \geq J^{\mathrm{FP}}(n, t)$, we obtain the desired result.

We note that the bound in (4) is also reported in [6] for the case when $\lambda^{*} t>n$. We show in Proposition 1 that this bound prevails when $\lambda^{*} t \leq n$. Note also that, while the bound in (3) is useful in showing the asymptotical optimality of FP and OFP heuristics, it can be arbitrarily small and cannot be used in our analysis.

The bound in Proposition 1 provides a performance guarantee of $1-e^{-1} \approx 0.6321$ for $n=1$, and this guarantee increases as $n$ increases. Therefore, the performance of the optimal fixed price heuristic (and the fixed price heuristic) is at least 0.6321 of what could be obtained through optimal dynamic pricing for any inventory pricing problem.

In the remainder of the paper, we focus on the case where the initial inventory is 1 . While the performance guarantee of 0.6321 is general, it is a loose bound. By characterizing the expected revenue of the optimal fixed price heuristic and the optimal dynamic pricing, we will obtain tight bounds for the optimal fixed price heuristic.

When the starting inventory is equal to 1 , the expected revenue of using a fixed demand rate $\lambda$ can be written as

$J^{f}(1, t, \lambda)=p(\lambda)\left(1-e^{-\lambda t}\right)$.

The next proposition states that $f^{f}(1, t, \lambda)$ given in (6) is unimodal in $\lambda$ for a given $t$ if the elasticity $\varepsilon(\lambda)=-p(\lambda) /\left(\lambda p^{\prime}(\lambda)\right)$ is decreasing in $\lambda$.

Proposition 2. $J^{f}(1, t, \lambda)$ is unimodal in $\lambda$ if $\varepsilon(\lambda)$ is decreasing in $\lambda$.

Proof. The derivative of the expected revenue with respect to $\lambda$ can be written as

$$
\begin{aligned}
\frac{\partial J^{f}(1, t, \lambda)}{\partial \lambda} & =p^{\prime}(\lambda)\left(1-e^{-\lambda t}\right)+t e^{-\lambda t} p(\lambda) \\
& =p^{\prime}(\lambda) e^{-\lambda t}\left(e^{\lambda t}-1-\varepsilon(\lambda) \lambda t\right) .
\end{aligned}
$$

Then, we get

$\frac{\partial J^{f}(1, t, \lambda)}{\partial \lambda}=p^{\prime}(\lambda) e^{-\lambda t} \lambda t\left(\frac{e^{\lambda t}-1}{\lambda t}-\varepsilon(\lambda)\right)$.

The first function in the parentheses in (7) is strictly increasing in $\lambda$. If the second function, $\varepsilon(\lambda)$, is a decreasing function, they intersect at only one point, say at $\lambda^{0}$. Therefore, $J^{f}(1, t ; \lambda)$ is increasing in $\lambda$ when $\lambda<\lambda^{0}$ and decreasing in $\lambda$ when $\lambda>\lambda^{o}$.

The condition in Proposition 2 is equivalent to price elasticity being increasing in price since the demand is decreasing in price. Note also that this is a sufficient condition, and it is one of the three most commonly used assumptions in revenue management literature (see [13]). Many commonly used price-response functions, including constant-elasticity, linear, exponential, and logit functions, satisfy this condition.

If $\varepsilon(\lambda)$ is decreasing in $\lambda$, the optimal fixed intensity $\lambda^{\circ}$ can be found by using the first-order condition which sets (7) to zero, leading to

$1+\varepsilon\left(\lambda^{o}\right) \lambda^{o} t=e^{\lambda^{o} t}$

\section{Specific price-response functions}

\subsection{Constant-elasticity price-response function}

We first consider the constant-elasticity price-response function $\lambda(p)=a p^{-\varepsilon}$, which is widely used in economics and marketing literature. The inverse of this function is $p(\lambda)=\left(\frac{a}{\lambda}\right)^{1 / \varepsilon}$, and the revenue rate as a function of demand rate is given by $r(\lambda)=\lambda\left(\frac{a}{\lambda}\right)^{1 / \varepsilon}$. Solving the differential equation in (1) for $n=1$ (see also [8]), one gets

$J^{*}(1, t)=(a t)^{1 / \varepsilon}\left(\frac{\varepsilon-1}{\varepsilon}\right)^{\frac{\varepsilon-1}{\varepsilon}}$.

Since the elasticity is constant (not increasing in $\lambda$ ), one can use the first-order condition

$1+\varepsilon \lambda^{o} t=e^{\lambda^{0} t}$

to obtain the optimal fixed intensity $\lambda^{0}$. Then the expected revenue of the optimal fixed price heuristic is

$J^{\text {OFP }}(1, t)=p\left(\lambda^{o}\right)\left(1-e^{-\lambda^{o} t}\right)=\left(\frac{a}{\lambda^{0}}\right)^{1 / \varepsilon}\left(1-e^{-\lambda^{o} t}\right)$,

where $\lambda^{0}$ satisfies (9).

The next proposition states that the smallest value of $J^{\mathrm{OFP}}(1, t) /$ $J^{*}(1, t)$ is approximately 0.8985 .

Proposition 3. Under a constant-price elasticity price-response function, the performance of the optimal fixed price heuristic is independent of $a$ and $t$. The minimum value of $J^{\mathrm{OPP}}(1, t) / J^{*}(1, t)$ is approximately 0.8985 when $\varepsilon \approx 2.5603$.

Proof. Denoting $z=\lambda^{0} t$, we have

$J^{\mathrm{OFP}}(1, t)=\frac{\left(1-e^{-z}\right)(a t)^{1 / \varepsilon}}{z^{1 / \varepsilon}}$,

where $z$ is the solution to $1+z \varepsilon=e^{z}$. Then, we obtain the ratio as $\frac{J^{\mathrm{OFP}}(1, t)}{J^{*}(1, t)}=\frac{1-e^{-z}}{\left(\frac{\varepsilon-1}{\varepsilon}\right)^{\frac{\varepsilon-1}{\varepsilon}} z^{1 / \varepsilon}}$.

Substituting $\varepsilon=\frac{e^{z}-1}{z}$, we can rewrite the ratio as $J^{\mathrm{OFP}}(1, t) / J^{*}(1, t)$ $=g(z)$, where

$g(z)=\frac{1-e^{-z}}{z^{\frac{z}{e^{z}-1}}\left(\frac{e^{z}-z-1}{e^{z}-1}\right)^{\frac{e^{z}-z-1}{e^{z}-1}}}$.

Then we can show that

$g^{\prime}(z)=\frac{(\ln (m(z))-\ln (z)+1)\left(e^{-z}-z e^{z}+z+e^{z}-2\right) z^{-z /\left(e^{z}-1\right)} m(z)^{-m(z)}}{\left(e^{z}-1\right)^{2}}$,

where $m(z)=\frac{e^{z}-z-1}{e^{z}-1}$

One can also show that $g^{\prime}(z)=0$ has a unique solution for $z>0$, leading to unimodality of $g$ in $z$. Solving $g^{\prime}(z)=0$ is equivalent to solving

$\ln \left(\frac{e^{z}-z-1}{e^{z}-1}\right)-\ln (z)+1=0$,

or

$-e^{z+1}+e^{z} z+z(e-1)+e=0$,

leading to a unique minimizer $z^{0} \approx 1.6566$ or $\varepsilon^{0} \approx 2.5603$ with objective function value $g\left(z^{0}\right) \approx 0.8985$. 


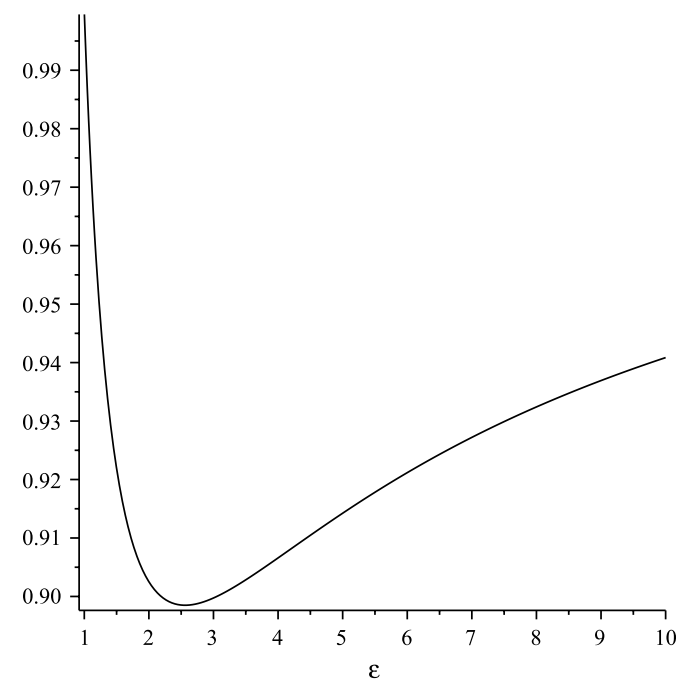

Fig. 1. Performance of optimal fixed pricing with a constant-elasticity price response function.

We note here that the suboptimality gap of $10.15 \%$ is larger than the largest gap reported so far in numerous computational studies in the literature for the optimal fixed pricing heuristic. Proposition 3 also states that the revenue impact of dynamic pricing depends only on the price elasticity of customers and is independent of the market size under a constant-elasticity priceresponse function. Fig. 1 shows the performance of the optimal fixed price heuristic as a function of price elasticity $\varepsilon$.

\subsection{Linear price-response function}

Now consider the linear price-response function, $\lambda(p)=a-b p$. The inverse of this function is $p(\lambda)=(a-\lambda) / b$. The elasticity for this function is given by $\varepsilon(\lambda)=(a-\lambda) / \lambda$, and the instantaneous revenue rate is given by $r(\lambda)=(a-\lambda) \lambda / b$. The linear priceresponse function is also widely used in the literature. This function is studied in detail in [7] as well. Solving the differential equation in (1), one gets

$J^{*}(1, t)=\frac{a^{2} t}{b(a t+4)}$.

Since the elasticity is increasing in $\lambda$, the first-order condition in (8) can be used to obtain the optimal fixed intensity. This leads to the equation

$\left(1+a t-\lambda^{o} t\right)=e^{\lambda^{o} t}$.

Then the expected revenue of the optimal fixed price heuristic is

$J^{\mathrm{OFP}}(1, t)=p\left(\lambda^{0}\right)\left(1-e^{-\lambda^{0} t}\right)=\frac{\left(a-\lambda^{0}\right)\left(1-e^{-\lambda^{0} t}\right)}{b}$,

where $\lambda^{0}$ satisfies (10).

The next proposition states that the smallest value of $J^{\mathrm{OFP}}(t) /$ $J^{*}(t)$ is approximately 0.9693 for the linear price-response function.

Proposition 4. Under a linear price-response function, the performance of the optimal fixed price heuristic depends only on the product at. The minimum value of $J^{\mathrm{OFP}}(1, t) / J^{*}(1, t)$ is approximately 0.9693 when at $\approx 16.39$.

Proof. The solution to (10) is $\lambda^{o}=\frac{a t+1-W\left(e^{a t+1}\right)}{t}$, where $W$ is the principal branch of the Lambert $W$ function. Using this we get

$J^{\mathrm{OFP}}(1, t)=\frac{\left(W\left(e^{a t+1}\right)-1\right)^{2}}{W\left(e^{a t+1}\right) b t}$.

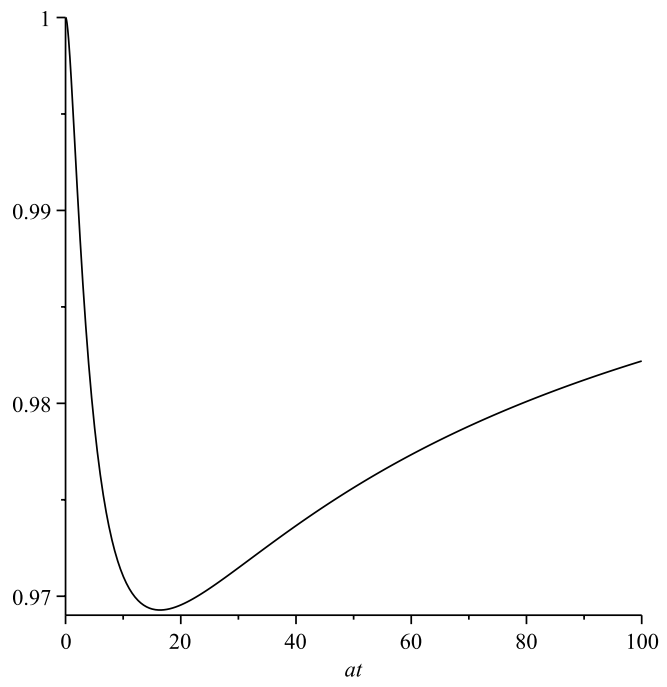

Fig. 2. Performance of optimal fixed pricing with a linear price response function.

Then we have

$\frac{J^{\mathrm{OFP}}(1, t)}{J^{*}(1, t)}=\frac{\left(W\left(e^{a t+1}\right)-1\right)^{2}(a t+4)}{W\left(e^{a t+1}\right) a^{2} t^{2}}$

We can denote this fraction as

$g(y)=\frac{\left(W\left(e^{y+1}\right)-1\right)^{2}(y+4)}{W\left(e^{y+1}\right) y^{2}}$,

where $y=a t$. Then we can find that

$g^{\prime}(y)=-\frac{\left(8 W\left(e^{y+1}\right)+y W\left(e^{y+1}\right)-y^{2}-5 y-8\right)\left(W\left(e^{y+1}\right)-1\right)}{W\left(e^{y+1}\right) y^{3}}$.

One can show that $g^{\prime}(y)=0$ has a unique solution for $y>0$, which leads to the unimodality of $g$ for $y>0$. Solving $g^{\prime}(y)=0$ for $y>0$, one gets the minimum of $g(y)$ as

$y^{o}=\frac{8 v}{4-v}$,

where $v$ satisfies

$-4 e^{v}+v e^{v}+v^{2}+3 v+4=0$.

This leads to the numerical values $y^{0} \approx 16.39$ and $g\left(y^{o}\right) \approx$ 0.9693 .

Note that at can be considered as a measure of the total market for the product since $a=\lambda(0)$ is the maximum demand rate and $t$ is the length of the horizon. Fig. 2 shows the performance of the optimal fixed price heuristic as a function of at.

We finally note again that constant-elasticity and linear priceresponse functions can be considered as the most favorable and least favorable scenarios for dynamic pricing. We numerically investigate the worst performance of optimal fixed pricing for other price-response functions. Our results show that the worst performance of the optimal fixed pricing heuristic under the exponential $\left(\log\right.$-lin) price-response function $\left(\lambda(p)=a e^{-p}\right)$ is $93.27 \%$ (confirming the observations in [6]). Under the logit price-response function $\left(\lambda(p)=a e^{-b p} /\left(1+e^{-b p}\right)\right)$, the worst performance is $93.38 \%$. Under the lin-log price-response function $(\lambda(p)=a-b \ln (p))$, the worst performance is $96.39 \%$. All of these bounds are within the bounds for constant-elasticity and linear price-response functions.

The theoretical bounds reported here have important implications for the use of dynamic pricing in the presence of inventory considerations. An opportunity to increase revenues by $10.15 \%$ through dynamic pricing is significant, particularly in industries that are appropriate for revenue management. On the other hand, 
if the price affects the demand in a linear fashion, the potential benefits of dynamic pricing are limited to only $3.07 \%$. The bounds that are developed here may also be useful for "full information" secretary problems where candidates' values are drawn from a known distribution.

\section{References}

[1] J.N. Bearden, R.O. Murphy, A. Rapoport, Decision biases in revenue management: some behavioral evidence, Manufacturing \& Service Operations Management 10 (2008) 625-636.

[2] G.R. Bitran, S.V. Mondschein, Periodic pricing of seasonal products in retailing, Management Science 43 (1997) 64-79.

[3] G. Elfving, A persistency problem connected with a point process, Journal of Applied Probability 4 (1967) 77-89.

[4] W. Elmaghraby, P. Keskinocak, Dynamic pricing in the presence of inventory considerations: research overview, current practices and future directions, Management Science 49 (2003) 1287-1309.
[5] P.R. Freeman, The secretary problem and its extensions: a review, International Statistical Review / Revue Internationale de Statistique 51 (1983) 189-206.

[6] G. Gallego, G. van Ryzin, Optimal dynamic pricing of inventories with stochastic demand over finite horizons, Management Science 40 (1994) 999-1020.

[7] W.M. Kincaid, D.A. Darling, An inventory pricing problem, Journal of Mathematical Analysis and Applications 7 (1963) 183-208.

[8] P.R. McAfee, V. te Velde, Dynamic pricing with constant demand elasticity, Production and Operations Management 17 (2008) 432-438.

[9] A. Sen, A comparison of fixed and dynamic pricing policies in revenue management, Omega 41 (2013) 586-597.

[10] W. Stadje, A full information pricing problem for the sale of several identical commodities, Mathematical Methods of Operations Research 34 (1990) 161-181.

[11] K. Talluri, G. van Ryzin, The Theory and Practice of Revenue Management, Kluwer Academic Publishers, 2004.

[12] W. Zhao, Y.-S. Zheng, Optimal dynamic pricing of perishable assets with nonhomogeneous demand, Management Science 46 (2000) 375-388.

[13] S. Ziya, H. Ayhan, R.D. Foley, Relationships among three assumptions in revenue management, Operations Research 52 (2004) 804-809. 